\title{
A Novel Orally Available Delta-5 Desaturase Inhibitor Prevents Atherosclerotic Lesions Accompanied by Changes in Fatty Acid Composition and Eicosanoid Production in ApoE Knockout Mice ${ }^{\mathbf{S}}$
}

\author{
Shuichi Takagahara, Hiromi Shinohara, Shigekazu Itokawa, Yoshinori Satomi, Ayumi Ando, \\ Takeshi Yamamoto, Hideo Suzuki, Takuya Fujimoto, Kazuki Kubo, and Shota Ikeda \\ Cardiovascular and Metabolic Drug Discovery Unit (S.T., H.Sh, S.I., T.Y., H.Su, T.F., K.K., S.I.) and Integrated Technology \\ Research Laboratories (Y.S., A.A.), Takeda Pharmaceutical Company Limited, Kanagawa, Japan
}

Received May 20, 2019; accepted August 30, 2019

\begin{abstract}
Delta-5 desaturase (D5D), encoded by fatty acid desaturase 1 (Fads1), is the rate-limiting enzyme for the conversion from dihomo- $\gamma$-linolenic acid (DGLA) to arachidonic acid (AA) in the $\omega-6$ polyunsaturated fatty acid pathway. Several AA-derived eicosanoids (e.g., prostaglandins, thromboxanes, and leukotrienes) and DGLA-derived eicosanoids are reported to promote and/or prevent atherosclerosis progression through, at least in part, its proinflammatory or anti-inflammatory effects. To elucidate the effects of D5D inhibition by a D5D inhibitor on atherosclerosis, we generated a potent, orally available and selective D5D inhibitor, 2-(2,2,3,3,3-Pentafluoropropoxy)-3-[4(2,2,2-trifluoroethoxy) phenyl]-5,7-dihydro-3H-pyrrolo[2,3-d]pyrimidine-4,6-dione, compound-326, and examined its effects on Western-diet fed $A p o E$ knockout (KO) mice. Oral administration of compound-326 (3-10 mg/kg per day for 15 weeks) significantly inhibited the progression of atherosclerotic lesions in the
\end{abstract}

aorta without affecting plasma total cholesterol and triglyceride levels. Compound-326 significantly decreased AA levels, while it increased DGLA levels in the liver and the blood accompanied by decreases in AA-derived eicosanoid production and increases in DGLA-derived eicosanoid production from the blood cells. We conclude that compound-326 prevents the progression of atherosclerosis in Western-diet fed $A p o E \mathrm{KO}$ mice by modulating a profile of eicosanoid production, suggesting that D5D inhibitors can be a novel remedy for preventing atherosclerosis and subsequent cardiovascular events.

\section{SIGNIFICANCE STATEMENT}

This study shows a D5D-specific and orally available potent inhibitor provided the first evidence to support the concept that D5D inhibitors will be a novel remedy for preventing the progression of atherosclerosis.

\section{Introduction}

Atherosclerosis and subsequent cardiovascular (CV) complications (including death, acute myocardial infarction, unstable angina, and stroke) are the leading causes of morbidity and mortality worldwide, in spite of major therapeutic progress in controlling classic risk factors, such as hypertension, dyslipidemia, and diabetes (Galkina and Ley, 2009; Arbab-Zadeh et al., 2012). Even when treated with powerful statins, some patients still have a high risk of CV complications after an acute coronary syndrome and stroke, suggesting an unmet need for new medications. One possibility that has strong potential is anti-inflammatory therapy. Several antiinflammatory drugs, such as darapladib (lipoprotein-associated phospholipase A2 inhibitor), varespladib (secreted phospholipases

This work was not supported by any grants. https://doi.org/10.1124/jpet.119.259846.

S This article has supplemental material available at jpet.aspetjournals.org.
A2 inhibitor), canakinumab (interleukin-1 $\beta$ monoclonal antibody), and low-dose methotrexate have been evaluated. Although some of the trials reported negative results (Mukherjee et al., 2001; Nicholls et al., 2014; O'Donoghue et al., 2014; White et al., 2014), anti-inflammatory therapy is still considered a promising strategy, since many clinical and preclinical studies suggest that inflammation plays a significant role in the development of atherosclerosis and subsequent complications (Ross, 1999; Duchatelle et al., 2012). In fact, it was recently reported that targeting the interleukin- $1 \beta$ pathway with canakinumab lead to significant prevention of CV events independent of lipid-level lowering in the CANTOS trial (Ridker et al., 2017), suggesting that anti-inflammatory therapy could be a new remedy for atherosclerosis and subsequent CV complications (Hansson, 2017).

The enzyme delta-5 desaturase (D5D) is encoded by the gene fatty acid desaturase 1 ( $F a d s 1$ ). This desaturase is the rate-limiting enzyme for polyunsaturated fatty acids (PUFA)

ABBREVIATIONS: AA, arachidonic acid; CV, cardiovascular; D5D, delta-5 desaturase; DGLA, dihomo- $\gamma$-linolenic acid; DHA, docosahexaenoic acid; EPA, eicosapentaenoic acid; 15-HETrE, 15-hydroxy eicosatrienoic acid; KO, knockout; LA, linoleic acid; LT, leukotriene; PG, prostaglandin; PUFA, polyunsaturated fatty acid; sICAM-1, soluble ICAM-1; TC, total cholesterol; TG, triglyceride; TX, thromboxane. 
conversion and determines systemic PUFA levels (Tosi et al., 2014). In the long-chain PUFA synthesis pathway, D5D catalyzes the conversion of dihomo- $\gamma$-linolenic acid (DGLA) into arachidonic acid [(AA), $\omega-6$ PUFA pathway] and the conversion of $\omega-3$ AA (20:4 $\omega$-3) into eicosapentaenoic acid [(EPA), $\omega$-3 pathway]. Several AA-derived eicosanoids including prostaglandins (PGs), thromboxanes (TXs), and leukotrienes (LTs) function as proinflammatory mediators (Capra et al., 2013), while some AA- and DGLA-derived eicosanoids including PGI2, PGE1, and 15-hydroxy eicosatrienoic acid (15-HETrE) have anti-inflammatory effects (Vang and Ziboh, 2005; Wang et al., 2012). These proinflammatory eicosanoids are reported to promote atherosclerosis, while inhibition of their synthesis showed antiatherosclerotic effects in rodents, rabbits, and pigs (Braun et al., 1993; Shen et al., 1996; Merhi et al., 1997; Cyrus et al., 2002; Kobayashi et al., 2004; Subbarao et al., 2004; Wang et al., 2006; Takai et al., 2009). These results suggest that modulation of the profile of AA- and DGLA-derived eicosanoids could affect atherosclerosis progression. In fact, Powell et al. (2016) and Fan et al. (2012) recently reported that Fads1 knockout (KO) mice showed systemic decreases in AA and AAderived prostaglandin amounts, increases in DGLA and DGLAderived prostaglandin amounts, and decreased atherosclerotic lesion formation in the aorta. These results suggest that D5D inhibition could be an attractive target for the treatment of atherosclerosis through its novel mechanism, despite the potential to induce developmental adaptations as one limitation of the genetic loss-of-function approach. To obtain proof-of-concept evidence that D5D inhibition by a selective D5D inhibitor has promise as a translatable therapy for atherosclerosis, it is important to develop a selective D5D inhibitor and to characterize its therapeutic efficacy in a preclinical animal model of atherosclerosis.

We previously reported that compound-326 (Suzuki et al., 2010), a newly discovered orally available, potent, and selective D5D inhibitor, ameliorated insulin resistance and obesity in a high fat diet-induced obese mouse model accompanied by alterations in the balance between DGLA and AA in the blood (Yashiro et al., 2016). In the present study, we evaluated the antiatherosclerotic effects of compound-326 in Western-diet fed ApoE KO mice. We examined the effects of the D5D inhibitor on the development of atherosclerosis, PUFA levels, eicosanoid production, and plasma levels of soluble ICAM-1 (sICAM-1), a systemic inflammatory marker, in this well-described animal model of atherosclerosis.

\section{Materials and Methods}

\section{Compound}

Compound-326 potently and selectively inhibits D5D activity, whereas it has almost no inhibitory effects on delta-6 and delta-9 desaturase activity (Yashiro et al., 2016). Its selectivity was investigated using a Cerep/PanLab selectivity profiling panel (Eurofins Discovery, St. Charles, MO) for 94 targets at $10 \mu \mathrm{M}$ of compound-326. The results are presented in Supplemental Table 1 as the percentage of inhibition of specific binding or activity.

\section{Animal Studies}

All laboratory animal care and protocols were approved by the Institutional Animal Care and Use Committee of the Takeda Pharmaceutical Company, and were conducted in conformity with the
Public Health Service Policy on Humane Care and Use of Laboratory Animals, incorporated in the Institute for Laboratory Animal Research Guide for Care and Use of Laboratory Animals. Supplemental Table 2 shows the fatty acid composition in the diets used in the study.

D5D Inhibitory Efficacy Evaluation Study Using Normal Mice. Male C57BL/6 mice (CLEA, Japan) were fed standard rodent chow (CE2; CLEA). Compound-326 [0.1-30 mg/kg per day, by mouth, suspended in $0.5 \%(\mathrm{w} / \mathrm{v})$ methylcellulose] was administered once daily to the mice via gavage for 1-4 days. Twenty-four hours after the final dosing, the mice were sacrificed and the livers were isolated. The levels of fatty acids in the liver were analyzed as detailed subsequently.

Antiatherosclerotic Study Using Fads1 $\times$ ApoE Double KO Mice. Fads1 KO mice were obtained from Lexicon Pharmaceuticals, Inc. (Powell et al., 2016) and Fads1 $\times$ ApoE double KO mice were generated by the Takeda $\mathrm{KO}$ animal generation group. Eightto 9 -week-old male Fads $1 \times A p o E$ double KO mice were fed the Western diet D12079B (Research Diets, New Brunswick, NJ) containing $21 \%$ fat and $0.15 \%$ cholesterol for 14 weeks. After 2 , 6,10 , and 14 weeks from the start of the Western diet, a blood sample was obtained via retro-orbital bleed, and assayed for biochemical parameters (fatty acid, total cholesterol, triglyceride, and glucose) analysis. At the end of the study period, mice were anesthetized by intraperitoneal administration of pentobarbital sodium, and the liver and the aorta were removed. The levels of fatty acids in the liver were analyzed as detailed subsequently. The isolated aortae, after removing extra tissues, were fixed with formalin and then stained with Oil Red O (Sigma, St. Louis, Mo.) to identify atherosclerotic lesions. The percentage of the lesion area was quantified with Image-Pro 6.0 software (Media Cybernetics, Rockville, MD).

Antiatherosclerosis Study Using Compound-326 (Studies 1 and 2).

Study 1. Male ApoE KO mice were used in this study. At 10 weeks of age, the mice were subjected to a control diet (98121701; Research Diets) for 1 week. At 11 weeks of age, the mice were then divided into five groups based on the baseline values of their body weight and plasma biochemical parameters (total cholesterol, triglyceride, and glucose in the nonfasting condition), followed by once-daily administration of compound$326(0.3,3$, and $10 \mathrm{mg} / \mathrm{kg}$ per day, by mouth) or vehicle $[0.5 \%(\mathrm{w} / \mathrm{v})$ methylcellulose] every morning via gavage. One week after the start of treatment, the mice were subjected to the Western diet for 14 weeks. One group of mice (administered with the same vehicle), as a control group, was maintained on the control diet. After 3, 7, 11 , and 15 weeks of compound-326 once-daily administration, blood samples were obtained by retro-orbital bleed and assayed for fatty acid composition and biochemical parameters (total cholesterol, triglyceride, and sICAM-1) analysis. At the end of the study period, the mice were sacrificed, and the blood, liver, and aorta were harvested. The atherosclerotic lesion area of the aorta was quantified as described previously in a blind manner.

Study 2. At 11 weeks of age, the mice were divided into six groups based on the baseline values of their body weight and plasma parameters (total cholesterol, triglyceride, glucose, and sICAM-1 levels in the nonfasting condition), followed by once-daily administration of compound-326 $(0.3,1,3$, and $10 \mathrm{mg} / \mathrm{kg}$ per day, by mouth) or vehicle as described previously. The other procedures were conducted as described in study 1 .

\section{Plasma Biochemical Parameter Analysis}

Plasma total cholesterol, triglyceride, and glucose levels were analyzed using an automatic chemistry analyzer (7180 Clinical Analyzer; Hitachi, Tokyo, Japan). Plasma sICAM-1 levels were assayed using the Mouse Soluble ICAM-1 (CD54) ELISA Kit (Pierce Biotechnology, Rockford, IL). 


\section{Eicosanoid Production Assay}

At the end of the study period, blood collected from the abdominal aorta was stimulated using $30 \mu \mathrm{M}$ A23187, 5-(Methylamino)-2-[[2R,3R,6S,8S,9R,11R)-3,9,11-trimethyl-8-[(1S)-1-methyl-2oxo-2-(1H-pyrrol-2-yl)-ethyl]-1,7-dioxaspiro[5.5]undec-2-yl]methyl]-4benzoxazolecarboxylic acid, and $10 \mu \mathrm{M} N$-formylmethionyl-leucylphenylalanine and incubated at $37^{\circ} \mathrm{C}$ for 10 minutes. The blood samples were then centrifuged at room temperature for 5 minutes to isolate the plasma. The plasma eicosanoid fraction was extracted using a solid-phase extraction column (Oasis HLB $30 \mathrm{mg} / \mathrm{cc}$; Waters, Japan). An aliquot ( $5 \mu \mathrm{l}$ ) of each sample was injected onto a UK-C18 reverse phase column $(150 \times 2 \mathrm{~mm}$; Imtakt, Japan $)$, and then chromatographic separation was performed by gradient elution of mobile phase A ( $0.1 \%$ acetic acid in Milli-Q, Millipore, Burlington, MA) and mobile phase $\mathrm{B}(0.1 \%$ acetic acid in acetonitrile). Eicosanoids (PGE2, TXB2, 11-dehydro-TXB2, LTB4, 6-keto-PGF1 $\alpha$, PGF2 $\alpha$, PGD2, 15-HETrE, and 13,14-dihydro-15-keto-PGE1) were measured by multiple reactions monitoring with a TSQ Vantage Triple Stage Quadrupole LC/MS Mass Spectrometer (Thermo Fisher Scientific Inc., San Jose, CA). Since we could not directly detect PGE1 and PGI2 production in blood cells by our method, we measured 13,14-dihydro-15-keto-PGE1 and 6-keto-PGF1 $\alpha$ production as surrogate markers of unstable PGE1 and PGI2, respectively.

\section{Quantitative Measurement of Fatty Acid Levels}

Total lipids in blood and liver samples were extracted with hexane/ 2-propanol/butylated hydroxytoluene (60:40:0.01, v/v). A liver phospholipid fraction was extracted using a solid-phase extraction column (Sep-Pak Vac NH2; Waters). Blood lipids and liver phospholipids were de-esterified and labeled with an esterified long-chain and short-chain fatty acid labeling reagent (YMC). The labeled fatty acid contents were measured via high-performance liquid chromatography (Agilent 1200; Agilent Technologies). In the antiatherosclerotic study using Fads1 $\mathrm{KO}$ mice, peak areas of AA to linoleic acid (LA) ratio (AA/LA) and DGLA to LA ratio (DGLA/LA) were quantified. In the D5D inhibitory efficacy evaluation study and the antiatherosclerosis study, each peak area of fatty acids was quantified and absolute amounts of fatty acids were quantified using margaric acid as an internal standard. The ratio of AA/DGLA was used to estimate D5D activity.

A

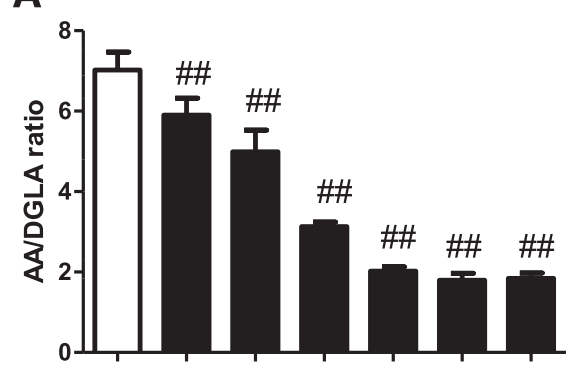

$\begin{array}{lllllll}\text { Vehicle } & 0.1 & 0.3 & 1 & 3 & 10 & 30\end{array}$

$(\mathrm{mg} / \mathrm{kg})$
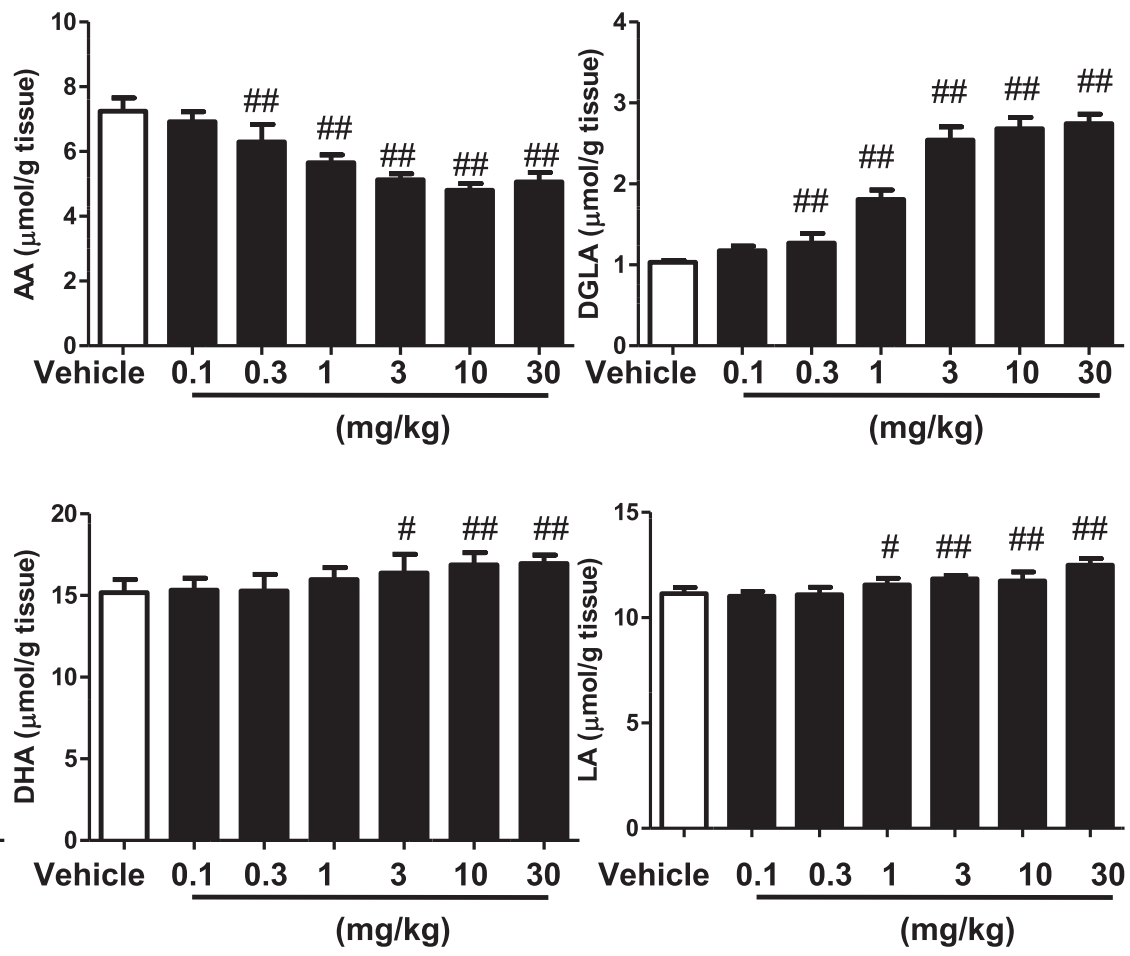

$(\mathrm{mg} / \mathrm{kg})$

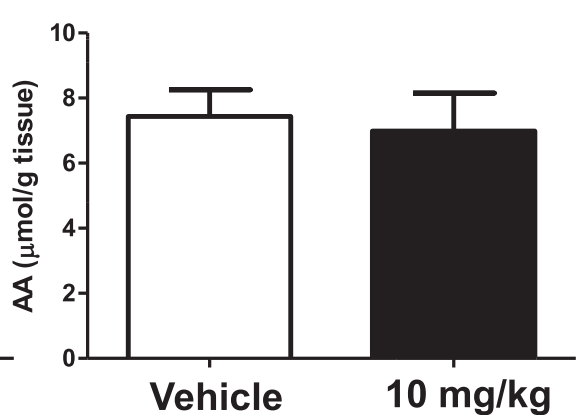

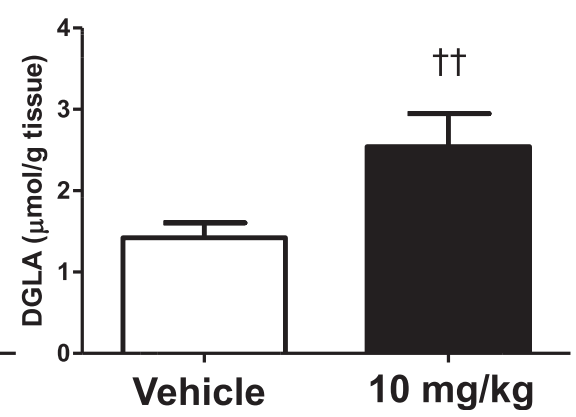

Fig. 1. The $\omega-6$ fatty acid levels from the livers of C57BL/6 mice fed a normal diet: (A) daily administration for four consecutive days; (B) single administration. Data are presented as mean \pm S.D. $(n=5)$. \#P $<0.025$; \#\#P<0.005 vs. vehicle by Williams test; $\dagger \dagger P<0.01$ vs. vehicle by AspinWelch test. 


\section{Data Analysis}

Data are expressed as mean \pm S.D. or mean \pm S.E.M. Statistical analysis was conducted in a step-by-step approach. A significant difference between control and vehicle-treated groups was assessed by Student's $t$ test or Aspin-Welch test depending on equal or unequal variances, and a value of $P<0.05$ was considered significant. The dose-dependent effect of compound-326 versus vehicle was then evaluated by the one-tailed Williams test or Shirley-Williams test depending on equal or unequal variances, and a value of $P<0.025$ was considered significant.

\section{Results}

D5D Inhibitory Efficacy Evaluation Study. Repeated administration of inhibitor compound-326 (at $0.1-30 \mathrm{mg} / \mathrm{kg}$ per day, by mouth, for 4 days) markedly and dosedependently decreased the AA/DGLA ratio and AA levels and increased the DGLA levels in the liver of normal mice. The effect of single administration showed moderate changes in the AA and DGLA levels in the liver compared with repeated administration (Fig. 1). It showed only moderate or no effects on other fatty acids including EPA and docosahexaenoic acid (DHA) in normal mice put on the normal diet (CE2), which richly contains $\omega-3$ PUFA (Supplemental Table 2).

Antiatherosclerotic Study Using Fads1 KO Mice. To determine the antiatherosclerotic effects of Fads1 gene deficiency, Fads1 homo KO, Fads1 hetero KO, and wild-type mice with an ApoE KO background were subjected to the Western diet for 14 weeks. Likewise, Powell et al. (2016) reported that Fads 1 homo KO mice showed significant prevention of aortic atherosclerosis lesion formation and body weight gaining, and changes in fatty acid compositions in tissues with no significant changes in plasma total cholesterol (TC) and triglyceride (TG) levels (Supplemental Figs. 1 and 2).

Antiatherosclerosis Study Using the D5D Inhibitor Compound-326 (Studies 1 and 2). To elucidate whether a D5D inhibitor could cause antiatherosclerotic effects as observed in Fads1 mutant mice, we administered compound-326 to ApoE KO mice fed the Western diet. We conducted two studies using two independent cohorts of mice to confirm the reproducibility of our results and a minimum effective dose. In both studies (studies 1 and 2), compound-326 (at $3-10 \mathrm{mg} / \mathrm{kg}$ per day, by mouth) showed statistically significant and reproducible prevention of aortic atherosclerosis lesion formation (Fig. 2).

Fatty acid levels were quantified in the liver and blood in test mice. Compound-326 dose-dependently decreased AA and DHA levels and increased DGLA levels in the liver, leading to a decreased AA/DGLA ratio (Fig. 3A). EPA levels in the liver were undetectable (the lower limit of detection: $0.2 \mu \mathrm{mol} / \mathrm{g}$ tissue) in mice who received compound-326 (3-10 mg/kg per day). In the blood, dosedependent decreases in AA, EPA, and DHA levels and increases in DGLA levels were observed throughout the study period (Fig. 3B). In addition, similar fatty acid changes were also observed in study 1 (Supplemental Fig. $3, \mathrm{~A}$ and $\mathrm{B})$.

To elucidate the antiatherosclerotic mechanism, eicosanoid production was measured in the blood of vehicle- and compound-326-treated ApoE KO mice. Figure 4 shows the results of blood samples taken from study 2. Compound-326 dose-dependently decreased the A23187, 5-(Methylamino)-2[[2R,3R,6S, 8S,9R,11R)-3,9,11-trimethyl-8-[(1S)-1-methyl-2oxo-2-(1H-pyrrol-2-yl)-ethyl]-1,7-dioxaspiro[5.5]undec-2-yl]methyl]4-benzoxazolecarboxylic acid, and $N$-formylmethionylleucyl-phenylalanine-stimulated production of AA-derived eicosanoids (PGE2, TXB2, LTB4, and 6-keto-PGF1 $\alpha$ ), while the DGLA-derived eicosanoid production (15-HETrE and 13,14-dihydro-15-keto-PGE1) was dose-dependently increased by the treatment of compound-326. Other eicosanoids (11-dehydro-TXB2, PGF2 $\alpha$, and PGD2) are shown in Supplemental Fig. 4. The same results were observed in the samples from study 1 (Supplemental Fig. 5). Accompanied by the antiatherosclerotic effects, the plasma levels of sICAM-1 were modestly but statistically
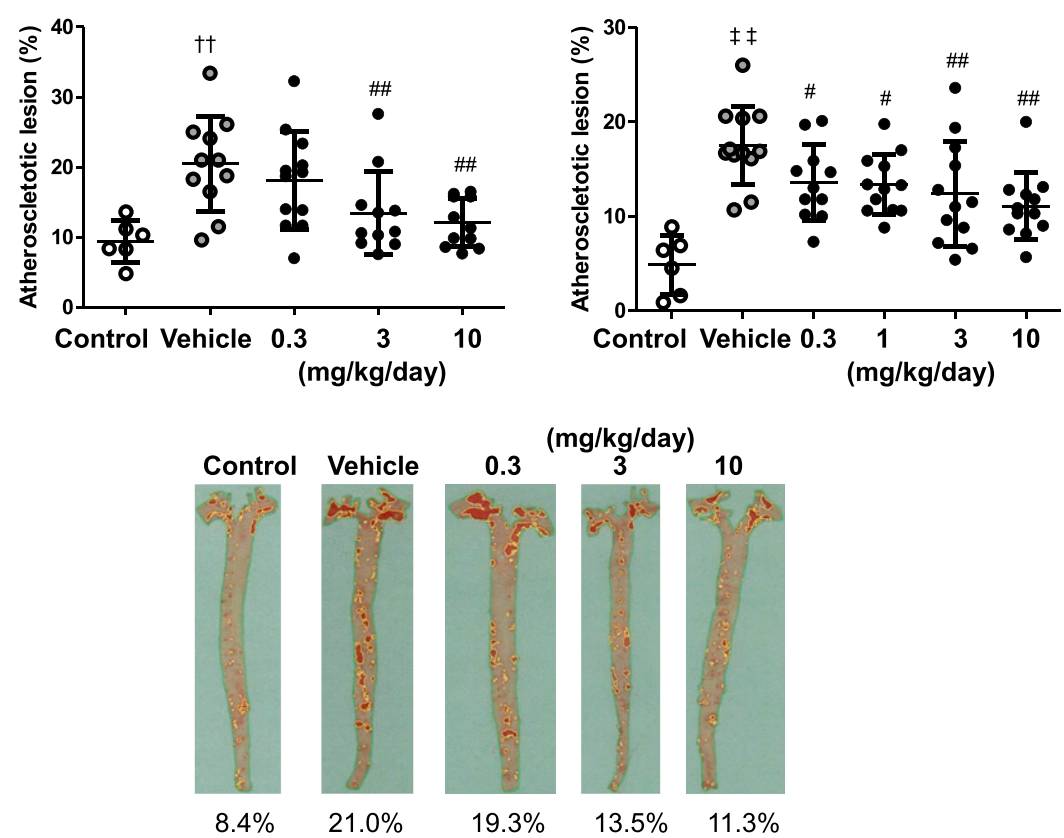

$(\mathrm{mg} / \mathrm{kg} / \mathrm{day})$

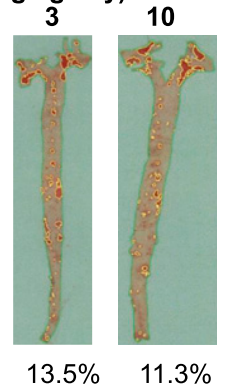

Fig. 2. Compound-326 administration inhibited the progression of atherosclerotic lesions in Western-diet fed ApoE KO mice (studies 1 and 2). After 15 weeks of compound-326 administration, aorta was harvested. The percentage of stained aortic area was quantified. Control mice were fed normal chow. The upper-left panel shows the results of study 1 . The number of mice in each group was as follows: control $(n=6)$, vehicle $(n=11), 0.3 \mathrm{mg} / \mathrm{kg}(n=12), 3 \mathrm{mg} / \mathrm{kg}$ $(n=11)$, and $10 \mathrm{mg} / \mathrm{kg}(n=11)$. The upper-right panel shows the results of study 2 . The number of mice in each group was as follows: control $(n=6)$, vehicle $(n=12)$, $0.3 \mathrm{mg} / \mathrm{kg}(n=12), 1 \mathrm{mg} / \mathrm{kg}(n=11), 3 \mathrm{mg} / \mathrm{kg}(n=12)$, and $10 \mathrm{mg} / \mathrm{kg}(n=12)$. Data are presented as mean \pm S.D. Representative images of each group are presented. The values of the percentage of lesions are described below the images. $\uparrow+P<0.01$ vs. control by Aspin-Welch test; $\$ \ddagger P<0.01$ vs. control by Student's $t$ test; $\# P<0.025$; $\# \# P<0.005$ vs. vehicle by Williams test. 
A
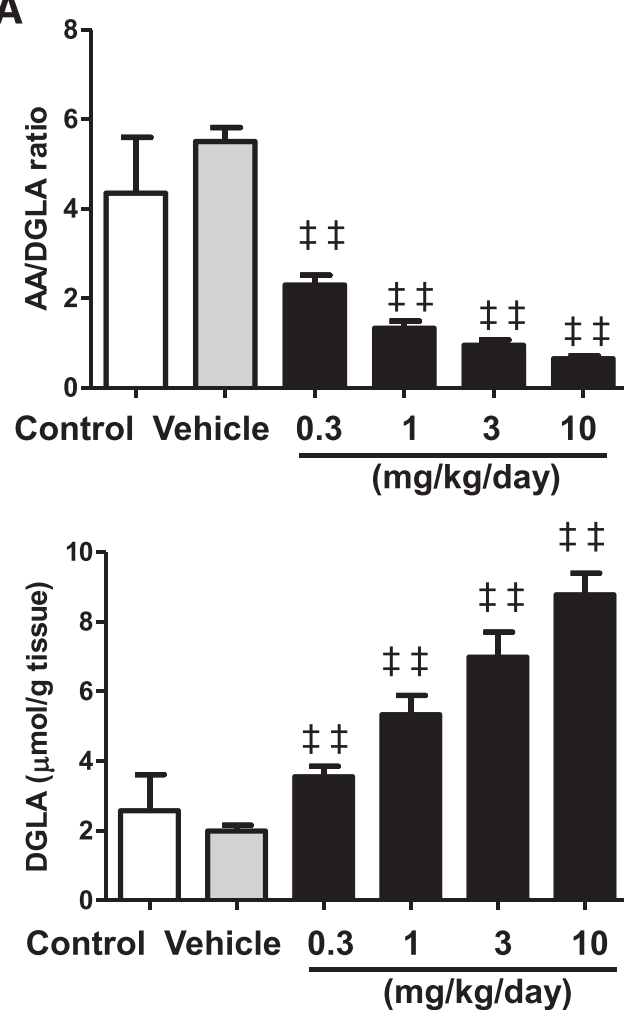

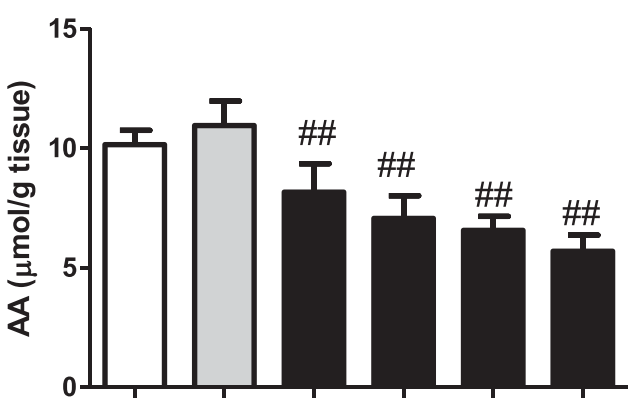

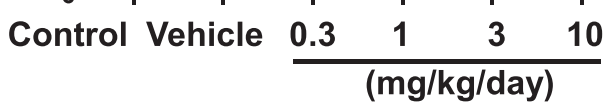

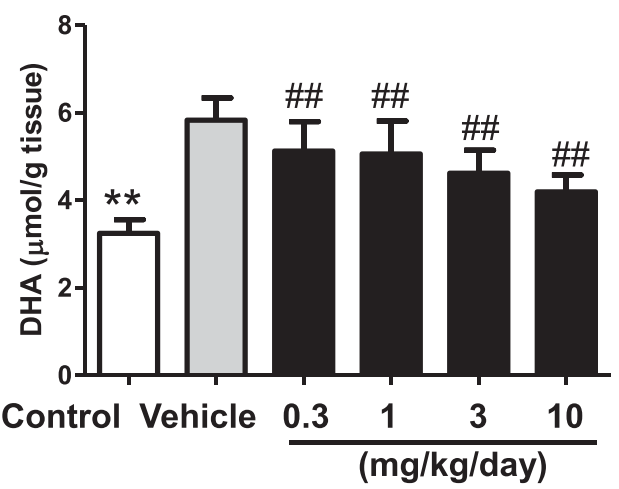

B
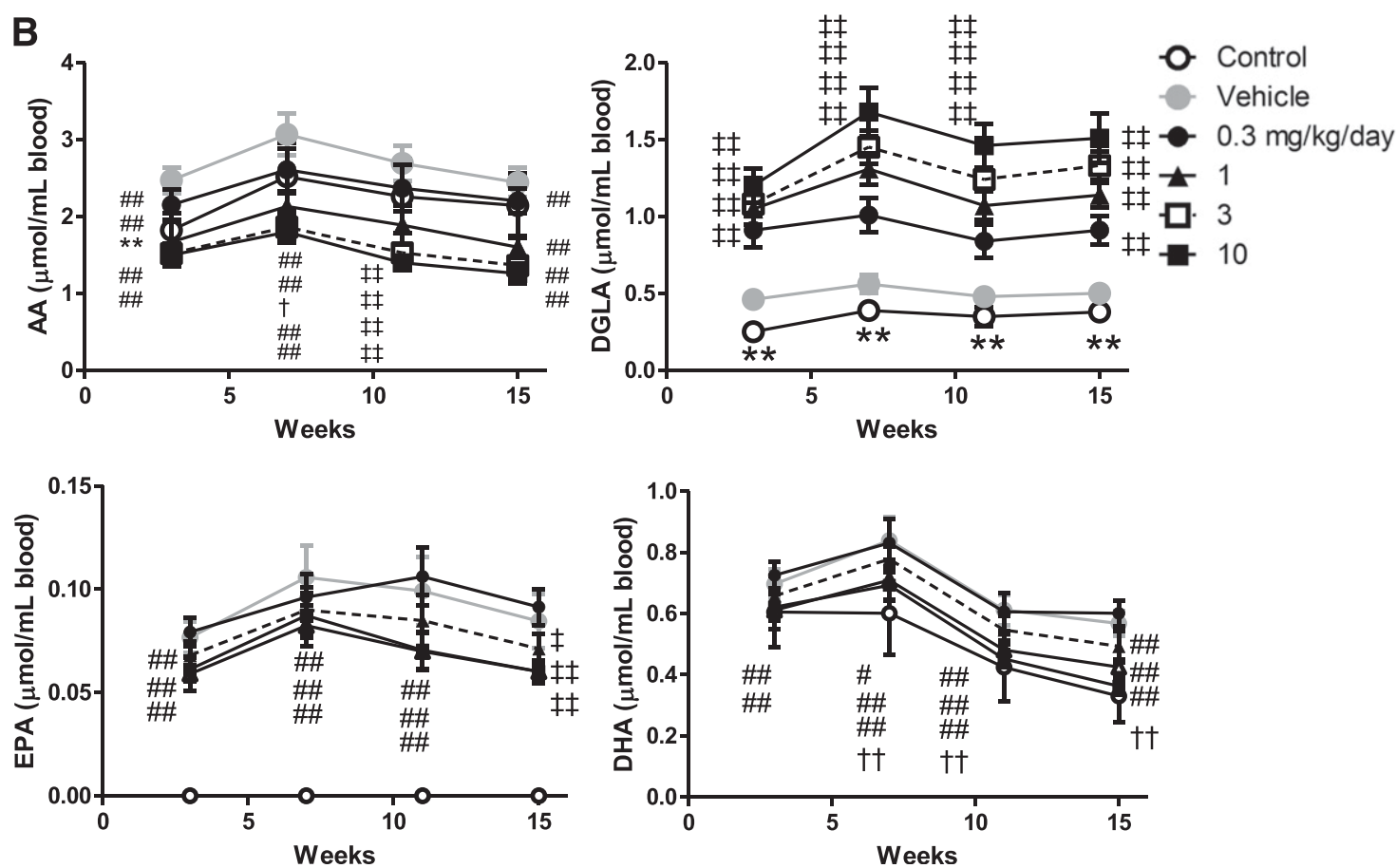

Fig. 3. The $\omega-6$ and $\omega-3$ fatty acid levels from ApoE KO mouse liver and blood administered with compound-326 (study 2): fatty acid analysis of mouse liver (A) and mouse blood (B). After 15 weeks of compound-326 administration, livers were harvested. The number of mice in each group is described in the legend of Fig. 2. The EPA levels in the blood from the control were undetectable. Data are presented as mean \pm S.D. $* * P<0.01$ vs. vehicle by Student's $t$ test; \#\#P<0.005 vs. vehicle by Williams test; $\uparrow P<0.05 ; \dagger \dagger P<0.01$ vs. vehicle by Aspin-Welch test; $\ddagger P<0.025 ; \ddagger \ddagger P<0.005$ vs. vehicle by Shirley-Williams test.

and significantly decreased by compound-326 administration at some points during the study period (Supplemental Fig. 6).

During the study period, body weight and the time course of plasma TC and TG levels were monitored. Plasma
TC and TG levels were not robustly altered by compound326 administration (Fig. 5). As we previously reported (Yashiro et al., 2016), compound-326-treated mice were dose-dependently leaner than the vehicle-treated mice (Fig. 6). 

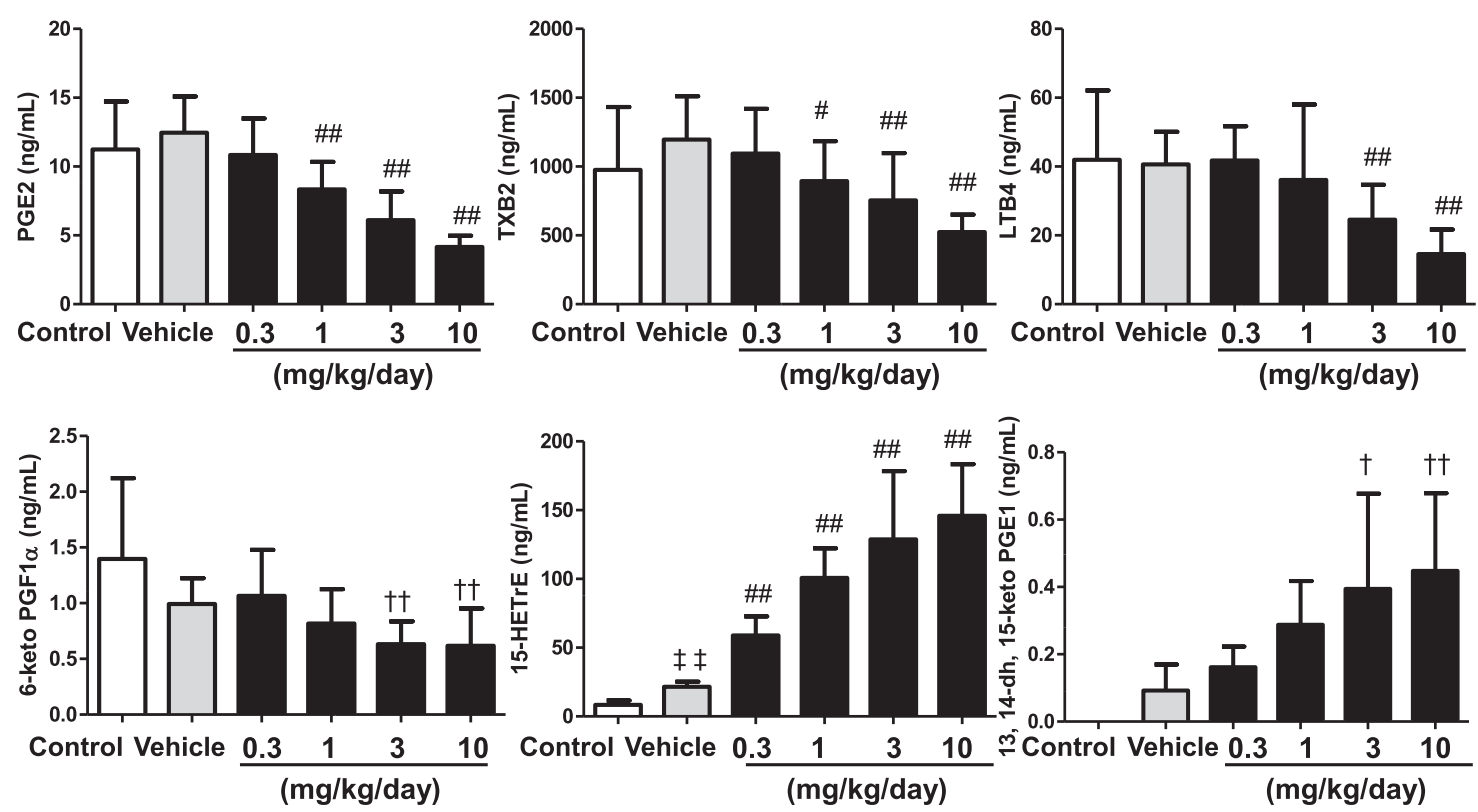

Fig. 4. Eicosanoid production from ApoE KO mouse blood stimulated with A23187,5-(Methylamino)-2-[[2R,3R,6S,8S,9R,11R)-3,9,11-trimethyl-8-[(1S)1-methyl-2-oxo-2-(1H-pyrrol-2-yl)-ethyl]-1,7-dioxaspiro[5.5] undec-2-yl]methyl]-4-benzoxazolecarboxylic acid, and $N$-formylmethionyl-leucyl-phenylalanine (fMLP) (study 2). ApoE KO mouse blood was stimulated with A23187 (30 $\mu \mathrm{M})$ and fMLP $(10 \mu \mathrm{M})$. Both reagents were added to blood samples at the same time. The number of mice in each group is described in the legend of Fig. 2 . Some samples (control, $n=6$; vehicle, $n=6 ; 0.3 \mathrm{mg} / \mathrm{kg}, n=5$ ) were removed from the data because the levels of 13,14-dihydro and 15-keto-PGE1 were not detectable. Data are presented as mean \pm S.D. $\ddagger \ddagger P<0.01$ vs. control by Student's $t$ test; $\# P<0.025$; \#\#P<0.005 vs. vehicle by Shirley-Williams test; $\uparrow P<0.025 ; \dagger \uparrow P<0.005$ vs. vehicle by Williams test. LTB4, leukotriene B4; TXB2, thromboxane B2.

\section{Discussion}

In the present study, we provide proof-of-concept evidence that D5D inhibition by a selective D5D inhibitor has promise as a translatable therapy for the treatment of atherosclerosis. Fan et al. (2012) and Powell et al. (2016) reported that Fads1 KO mice showed a decrease in AA levels and an increase in DGLA levels in colon mucosa, liver, splenocytes, brain, and serum accompanied by a decrease in PGE2 levels, an increase in PGE1 levels in tissues, and a decrease of atherosclerotic lesion formation in the aorta (Fan et al., 2012; Powell et al., 2016). Although these results clearly suggest the value of D5D as a promising target for atherosclerosis, it is important to obtain proof-of-concept data with a specific D5D inhibitor to show the clinical feasibility and translatability. We provide three critical lines of evidence utilizing a potent, orally available and selective D5D inhibitor, compound-326. First, 4-day oral treatment with compound-326 was enough to induce significant changes in AA and DGLA levels in normal mice in a dose-dependent manner. Second, the administration of compound-326 also showed significant alterations of this fatty acid composition in Western-diet fed ApoE KO mice accompanied by modulating profiles of eicosanoid production in blood cells. Finally, compound-326 significantly prevented aortic atherosclerosis lesion formation in the two independent cohorts of mice. These results provide proof-of-concept evidence that D5D inhibition by a selective D5D inhibitor represents a promising potential therapy for atherosclerosis.

Several lines of previous work suggest that the mechanism of the antiatherosclerosis efficacy of D5D inhibition may be related to the change in the profile of eicosanoid production and inflammatory pathway modulation through alterations of AA and DGLA levels. Several reports suggest the unique roles of AA-derived eicosanoids in the progression of atherosclerosis by utilizing low-dose aspirin administration, PGE2 synthase enzyme ( $m P G E S-1)$ deletion, LTB4 receptor (BLT-1) deletion, thromboxane receptor (TP) deletion, and PGI receptor (IP) deletion in mice (Cyrus et al., 2002; Kobayashi et al., 2004; Subbarao et al., 2004; Wang et al., 2006). Based on the crucial role of inflammation in the pathogenesis of atherosclerosis, and the well-described inflammatory signaling pathways of these eicosanoids, the mechanism of antiatherosclerosis effects observed in the present mouse studies is considered to be, at least in part, related to its inflammatory modulation. In the present study, we found compound-326 showed a weak but statistically significant decrease in a systemic inflammation marker, sICAM-1, in plasma at some points during the study period, in agreement with the relationship between its antiatherosclerosis effects.

As Powell et al. (2016) reported, we confirmed that genetic D5D deficiency could prevent the progression of atherosclerotic lesions in Western-diet fed Fads1 homo KO $\times A p o E$ KO mice. Since Fads 1 hetero KO mice did not show a decrease in atherosclerotic lesions in the aorta, it is likely that $50 \%$ inhibition of the enzyme accompanied by a slight change in AA and DGLA levels (less than 10\% decrease in the AA/LA ratio and less than $40 \%$ increase in the DGLA/LA ratio in blood cells) was not enough to show antiatherosclerotic effects. The antiatherosclerotic effects of compound-326 were observed in more than $30 \%$ of the decreases in the AA levels and more than $120 \%$ of the increases in DGLA levels in blood cells, suggesting there is a threshold of arachinodante for showing antiatherosclerotic effects. D5D inhibition showed a significant decrease among AA-derived eicosanoids in both proinflammatory eicosanoids and a metabolite of anti-inflammatory eicosanoid PGI2, 6-keto-PGF1 $\alpha$, and $\omega$-3 fatty acid (EPA and DHA) levels, which have been reported to show antiatherosclerotic 
A
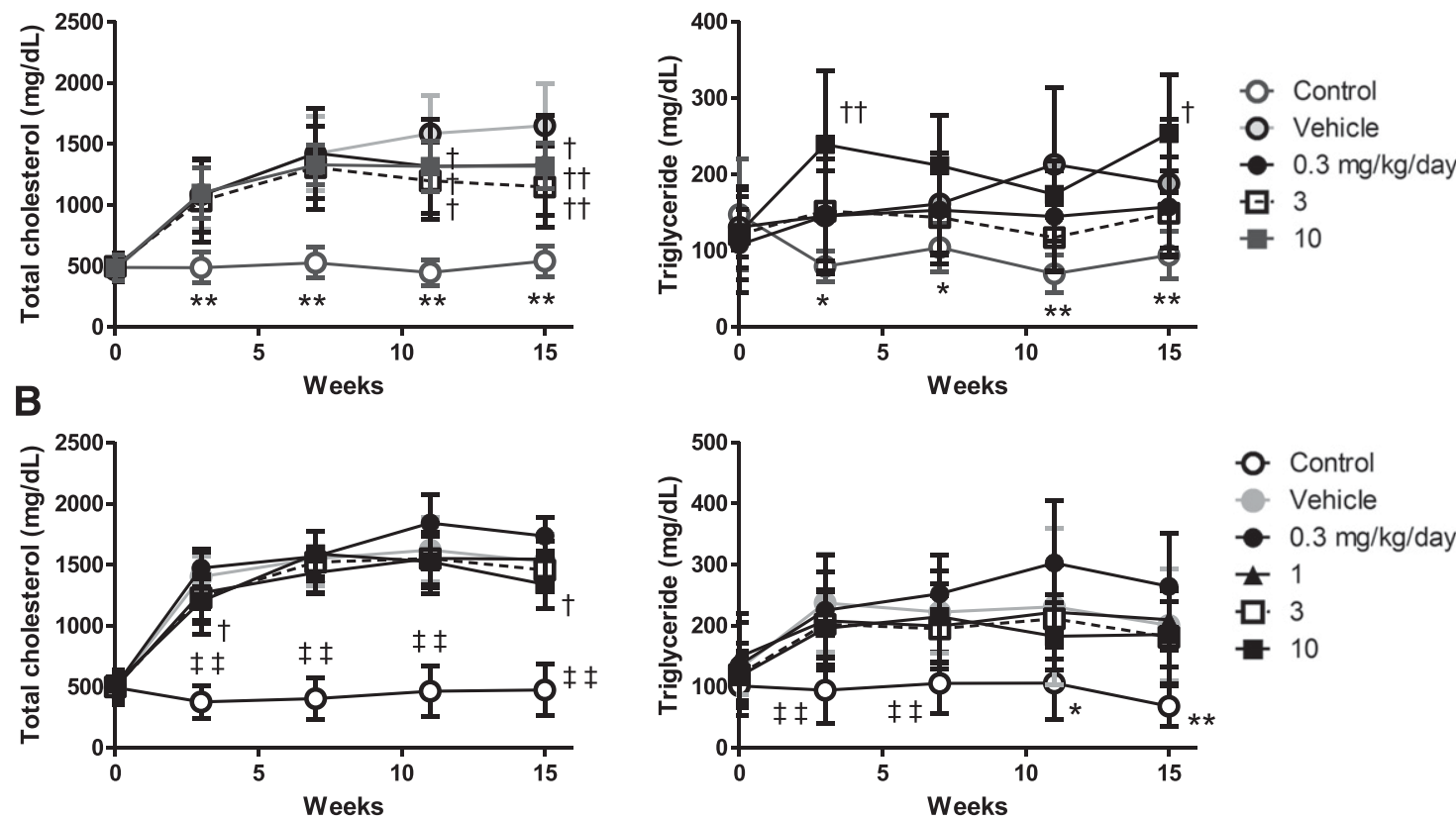

Fig. 5. Effects of compound-326 on plasma total cholesterol and triglyceride levels (studies 1 and 2). Time course of plasma total cholesterol and triglyceride levels. Plasma was obtained at $0,3,7,11$, and 15 weeks of the study period; study 1 (A) and study 2 (B). The number of mice in each group is described in the legend of Fig. 2. Data are presented as mean \pm S.D. ${ }^{*} P<0.05 ;{ }^{*} P<0.01$ vs. vehicle by Aspin-Welch test; $\$ \neq P<0.01$ vs. control by $t$ test; $\dagger P<0.025$ vs. vehicle by Williams test; $\dagger \dagger P<0.005$ vs. vehicle by Williams test.

effects through anti-inflammatory mechanisms in mouse models (Matsumoto et al., 2008; Wan et al., 2010). The details of its mechanism should be examined, but we speculate that an increase in DGLA-derived anti-inflammatory eicosanoid (e.g., PGE1 and 15-HETrE) production and a decrease in several AA-derived inflammatory eicosanoids (e.g., PGE2, LTB4 and TXB2) simultaneously could overcome the negative effects of a decrease in PGI2 and $\omega$-3 fatty acid levels (Vang and Ziboh, 2005; Wang et al., 2012).

Compound-326 showed minimum effects on plasma TC and TG levels in ApoE KO mice fed the Western diet. This suggests that its antiatherosclerotic effects are independent of cholesterollowering effects. Hypercholesterolemia is an important factor in the progression of atherosclerotic lesions, and drugs that decrease cholesterol levels reduce $\mathrm{CV}$ events in the clinical setting. The present results may suggest the usefulness of combination therapy with a D5D inhibitor and cholesterol-lowering drugs for the prevention of $\mathrm{CV}$ events.

We observed body weight loss in both Fads1 $\times$ ApoE double KO mice and compound-326-treated $A p o E$ KO mice fed the Western diet. Since a difference in body weight between wild and Fads 1 homo KO mice was not observed under normal
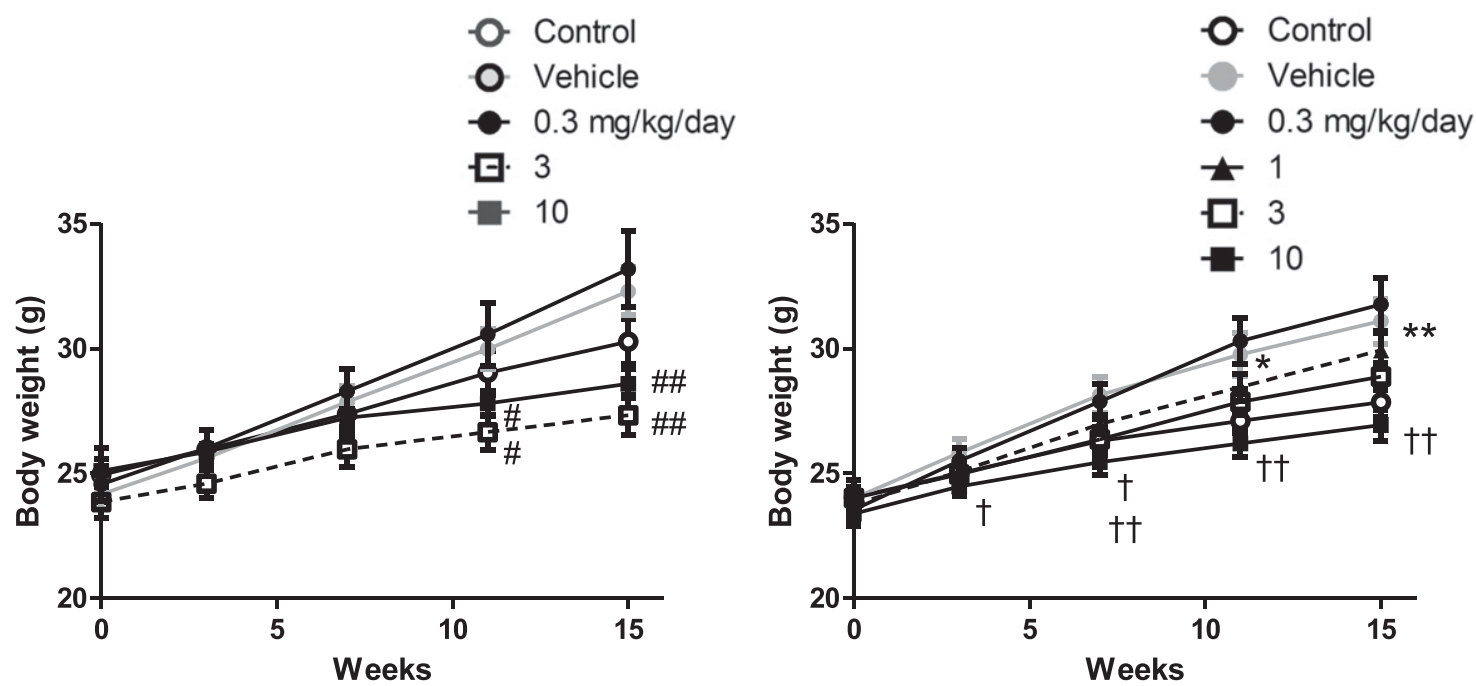

Fig. 6. Effects of compound-326 on body weight (studies 1 and 2). The left and right panels show the results of studies 1 and 2 , respectively. The number of mice in each group is described in the legend of Fig. 1. Data are presented as mean \pm S.E.M. $* P<0.05 ; * * P<0.01$ vs. control by Aspin-Welch test; $\# P<0.025 ; \# \# P<0.005$ vs. vehicle by Shirley-Williams test; $\uparrow P<0.025 ; \dagger \dagger P<0.005$ vs. vehicle by Williams test. 
chow fed conditions, it is likely that D5D inhibition leads to reduction of fat mass gain. Powell et al. (2016) reported reduction of fat mass in Fads1 $\times$ ApoE double KO mice fed both high fat and Western diets with minimum effects on lean body mass. We also reported that compound-326 lowered insulin resistance and body weight gain accompanied by a decrease in macrophage infiltration into adipose tissue in a diet-induced obese mouse model (Yashiro et al., 2016). These results are consistent with the present results. Although one might be interested in the interaction between D5D inhibition-induced antiobesity and antiatherosclerotic effects, its detailed mechanism of action remains to be elucidated.

Our study has some limitations. First, the characteristics of atherosclerotic lesions in the ApoE $\mathrm{KO}$ mice we used can differ considerably from those of human unstable atherosclerotic plaques. Although inflammation plays an important role in pathogenesis of both settings, human unstable plaques are much more severe and can progress to plaque rupture, unlike those in mice. Therefore, it is important to clarify whether D5D inhibition is effective in preventing rupture in unstable atherosclerosis or an atherosclerosis preexisting model. If so, D5D inhibition is expected to show benefits in the secondary prevention of myocardial infarction. Second, more research is needed to compare the effects of D5D inhibition and other antiatherosclerosis strategies, such as lipid-lowering drugs or anti-inflammatory drugs. Although several inflammatory enzymes (lipoprotein-associated phospholipase A2, secreted phospholipases A2, COX-2, etc.), highly expressed in atherosclerotic lesions, produce proinflammatory and proapoptotic mediators, these enzyme inhibitors have not shown promising results in clinical studies (Mukherjee et al., 2001; Nicholls et al., 2014; O'Donoghue et al., 2014; White et al., 2014). Further studies are needed to better understand the detailed mechanism of action that can relate D5D inhibition to its antiatherosclerotic effects. We think it is interesting to conduct in vivo and in vitro studies such as analysis of inflammatory cell infiltrations and macrophage activity to test the hypothesis that D5D inhibition has anti-inflammatory effects. Finally, we need to clarify potential adverse effects by D5D inhibition, especially in the central nervous system. We identified that D5D inhibition caused a reduction in AA levels in the brain (Supplemental Fig. 7); AA is the substrate for the synthesis of endocannabinoids, which mediate their endocannabinoid receptor, CB1, signaling. Signal alterations by CB1 antagonist treatments have been reported to lead to psychiatric adverse effects including depression (Leite et al., 2009). It would be critical to clarify if D5D inhibition can cause neuropsychiatric effects.

In summary, in the present study we identified a D5Dspecific and orally available potent inhibitor and provided the first evidence to support the concept that D5D inhibitors will be a novel remedy for preventing atherosclerosis and subsequent CV events through its novel mechanism.

\section{Acknowledgments}

We thank Makiko Itasaka and Masatoshi Yoshimura for excellent technical assistance. We also thank Hidenori Kamiguchi for helpful discussions.

\section{Authorship Contributions}

Participated in research design: Takagahara, Itokawa, Kubo, Ikeda.
Conducted experiments: Yamamoto, Suzuki, Fujimoto, Takagahara, Shinohara, Itokawa.

Performed data analysis: Takagahara, Shinohara, Satomi, Ando, Ikeda.

Wrote or contributed to the writing of the manuscript: Takagahara, Ikeda.

\section{References}

Arbab-Zadeh A, Nakano M, Virmani R, and Fuster V (2012) Acute coronary events. Circulation 125:1147-1156.

Braun M, Hohlfeld T, Kienbaum P, Weber AA, Sarbia M, and Schrör K (1993) Antiatherosclerotic effects of oral cicaprost in experimental hypercholesterolemia in rabbits. Atherosclerosis 103:93-105.

Capra V, Bäck M, Barbieri SS, Camera M, Tremoli E, and Rovati GE (2013) Eicosanoids and their drugs in cardiovascular diseases: focus on atherosclerosis and stroke. Med Res Rev 33:364-438.

Cyrus T, Sung S, Zhao L, Funk CD, Tang S, and Praticò D (2002) Effect of low-dose aspirin on vascular inflammation, plaque stability, and atherogenesis in lowdensity lipoprotein receptor-deficient mice. Circulation 106:1282-1287.

Duchatelle V, Kritikou EA, and Tardif JC (2012) Clinical value of drugs targeting inflammation for the management of coronary artery disease. Can $J$ Cardiol 28: 678-686.

Fan YY, Monk JM, Hou TY, Callway E, Vincent L, Weeks B, Yang P, and Chapkin RS (2012) Characterization of an arachidonic acid-deficient (Fads1 knockout) mouse model. J Lipid Res 53:1287-1295.

Galkina $\mathrm{E}$ and Ley K (2009) Immune and inflammatory mechanisms of atherosclerosis. Annu Rev Immunol 27:165-197.

Hansson GK (2017) Inflammation and atherosclerosis: the end of a controversy. Circulation 136:1875-1877.

Kobayashi T, Tahara Y, Matsumoto M, Iguchi M, Sano H, Murayama T, Arai H, Oida $\mathrm{H}$, Yurugi-Kobayashi T, Yamashita JK, et al. (2004) Roles of thromboxane $\mathrm{A}_{2}$ and prostacyclin in the development of atherosclerosis in apoE-deficient mice. J Clin Invest 114:784-794.

Leite CE, Mocelin CA, Petersen GO, Leal MB, and Thiesen FV (2009) Rimonabant: an antagonist drug of the endocannabinoid system for the treatment of obesity. Pharmacol Rep 61:217-224.

Matsumoto M, Sata M, Fukuda D, Tanaka K, Soma M, Hirata Y, and Nagai R (2008) Orally administered eicosapentaenoic acid reduces and stabilizes atherosclerotic lesions in ApoE-deficient mice. Atherosclerosis 197:524-533.

Merhi Y, Provost P, Guidoin R, and Latour JG (1997) Importance of platelets in neutrophil adhesion and vasoconstriction after deep carotid arterial injury by angioplasty in pigs. Arterioscler Thromb Vasc Biol 17:1185-1191.

Mukherjee D, Nissen SE, and Topol EJ (2001) Risk of cardiovascular events associated with selective COX-2 inhibitors. JAMA 286:954-959.

Nicholls SJ, Kastelein JJ, Schwartz GG, Bash D, Rosenson RS, Cavender MA, Brennan DM, Koenig W, Jukema JW, Nambi V, et al.; VISTA-16 Investigators (2014) Varespladib and cardiovascular events in patients with an acute coronary syndrome: the VISTA-16 randomized clinical trial. JAMA 311:252-262.

O'Donoghue ML, Braunwald E, White HD, Lukas MA, Tarka E, Steg PG, Hochman JS, Bode C, Maggioni AP, Im K, et al.; SOLID-TIMI 52 Investigators (2014) Effect of darapladib on major coronary events after an acute coronary syndrome: the SOLID-TIMI 52 randomized clinical trial [published correction appears in JAMA (2014) 312:1473]. JAMA 312:1006-1015.

Powell DR, Gay JP, Smith M, Wilganowski N, Harris A, Holland A, Reyes M, Kirkham L, Kirkpatrick LL, Zambrowicz B, et al. (2016) Fatty acid desaturase 1 knockout mice are lean with improved glycemic control and decreased development of atheromatous plaque. Diabetes Metab Syndr Obes 9:185-199.

Ridker PM, Everett BM, Thuren T, MacFadyen JG, Chang WH, Ballantyne C, Fonseca F, Nicolau J, Koenig W, Anker SD, et al.; CANTOS Trial Group (2017) Antiinflammatory therapy with canakinumab for atherosclerotic disease. $N \mathrm{Engl}$ J Med 377:1119-1131.

Ross R (1999) Atherosclerosis-an inflammatory disease. N Engl J Med 340:115-126. Shen J, Herderick E, Cornhill JF, Zsigmond E, Kim HS, Kühn H, Guevara NV, and Chan L (1996) Macrophage-mediated 15-lipoxygenase expression protects against atherosclerosis development. J Clin Invest 98:2201-2208.

Subbarao K, Jala VR, Mathis S, Suttles J, Zacharias W, Ahamed J, Ali H, Tseng MT, and Haribabu B (2004) Role of leukotriene $B_{4}$ receptors in the development of atherosclerosis: potential mechanisms. Arterioscler Thromb Vasc Biol 24:369-375.

Suzuki H, Fujimoto T, and Yamamoto T (2010) inventors, Takeda Pharmaceutical Co Ltd, assignee. Delta-5-desaturase inhibitors. Patent WO2010087467 A1. PCT application. 5 Aug 2010.

Takai S, Jin D, Kawashima H, Kimura M, Shiraishi-Tateishi A, Tanaka T, Kakutani S, Tanaka K, Kiso Y, and Miyazaki M (2009) Anti-atherosclerotic effects of dihomo$\gamma$-linolenic acid in apoE-deficient mice. $J$ Atheroscler Thromb 16:480-489.

Tosi F, Sartori F, Guarini P, Olivieri O, and Martinelli N (2014) Delta-5 and delta-6 desaturases: crucial enzymes in polyunsaturated fatty acid-related pathways with pleiotropic influences in health and disease. Adv Exp Med Biol 824:61-81.

Vang K and Ziboh VA (2005) 15-Lipoxygenase metabolites of $\gamma$-linolenic acid/eicosapentaenoic acid suppress growth and arachidonic acid metabolism in human prostatic adenocarcinoma cells: possible implications of dietary fatty acids. Prostaglandins Leukot Essent Fatty Acids 72:363-372.

Wan JB, Huang LL, Rong R, Tan R, Wang J, and Kang JX (2010) Endogenously decreasing tissue $\mathrm{n}-6 / \mathrm{n}-3$ fatty acid ratio reduces atherosclerotic lesions in apolipoprotein E-deficient mice by inhibiting systemic and vascular inflammation. Arterioscler Thromb Vasc Biol 30:2487-2494.

Wang M, Zukas AM, Hui Y, Ricciotti E, Puré E, and FitzGerald GA (2006) Deletion of microsomal prostaglandin E synthase-1 augments prostacyclin and retards atherogenesis. Proc Natl Acad Sci USA 103:14507-14512. 
Wang X, Lin H, and Gu Y (2012) Multiple roles of dihomo- $\gamma$-linolenic acid against proliferation diseases. Lipids Health Dis 11:25.

White HD, Held C, Stewart R, Tarka E, Brown R, Davies RY, Budaj A, Harrington RA, Steg PG, Ardissino D, et al.; STABILITY Investigators (2014) Darapladib for preventing ischemic events in stable coronary heart disease. $N$ Engl J Med 370: 1702-1711.

Yashiro H, Takagahara S, Tamura YO, Miyahisa I, Matsui J, Suzuki H, Ikeda S, and Watanabe M (2016) A novel selective inhibitor of delta-5 desaturase lowers insulin resistance and reduces body weight in diet-induced obese C57BL/6J mice. PLoS One 11:e0166198.

Address correspondence to: Shuichi Takagahara, Cardiovascular and Metabolic Drug Discovery Unit, Takeda Pharmaceutical Company Limited, Kanagawa 251-8555, Japan. E-mail: shuichi.takagahara@takeda.com 